\title{
A randomized algorithm for the on-line weighted bipartite matching problem *
}

\author{
Béla Csaba $^{\dagger} \quad$ András Pluhár ${ }^{\ddagger}$
}

\begin{abstract}
We study the on-line minimum weighted bipartite matching problem in arbitrary metric spaces. Here, $n$ not necessary disjoint points of a metric space $M$ are given, and are to be matched on-line with $n$ points of $M$ revealed one by one. The cost of a matching is the sum of the distances of the matched points, and the goal is to find or approximate its minimum. The competitive ratio of the deterministic problem is known to be $\Theta(n)$, see [7, 11]. It was conjectured in [8] that a randomized algorithm may perform better against an oblivious adversary, namely with an expected competitive ratio $\Theta(\log n)$. We prove a slightly weaker result by showing a $o\left(\log ^{3} n\right)$ upper bound on the expected competitive ratio. As an application the same upper bound holds for the notoriously hard fire station problem, where $M$ is the real line, see $[6,12]$.
\end{abstract}

Keywords: On-line, bipartite matching, randomized, metric spaces. 1991 Mathematics Subject Classification: 68R10,68W25, 68W40

\section{Introduction}

Finding a minimum weight matching in a weighted graph $G$ is a well studied problem in graph theory. Much less is known about its on-line version; here we briefly introduce the set-up and the most important results. For more thorough references see $[7,8$, $11,12]$.

Let $G$ be an arbitrary weighted graph, and given two players, $A$ and $B$, we consider the following on-line matching game on $G$ : First, $A$ picks the multiset $S=\left\{s_{1}, \ldots, s_{n}\right\}$ of $V(G)$, these are the servers. Then, one by one, $A$ discloses the requests, again a multiset $R=\left\{r_{1}, \ldots, r_{n}\right\}$ of $V(G)$. When an element of $R$ is requested, $B$ has to match it with some unmatched element from $S$, and $B$ wishes to minimize the cost of the resulted matching.

It is clear that usually $B$ cannot reach the offline minimum, and the competitive ratio, that is the online cost/offline optimum, is infinite if one has no further assumption on $G$ (see Kalyanasundaram and Pruhs, and Khuller et al in [7, 11]). It was

\footnotetext{
*The authors were partially supported by OTKA grants T034475 and T049398.

${ }^{\dagger}$ Analysis and Stochastics Research Group of the Hungarian Academy of Sciences, Szeged, Aradi vértanúk tere 1, H-6720. email: bcsaba@math.u-szeged.hu

${ }^{\ddagger}$ Department of Computer Science, University of Szeged, Szeged, Árpád tér 2., H-6720. email: pluhar@inf.u-szeged.hu
} 
assumed in both papers that the weights are nonnegative, and satisfy the triangle inequality, so one may refer to the graph $G$ as a metric space $M=(X, d)$ with underlying set $X$ and distance function $d$, while the multisets $S$ and $R$ are repeated points of $M$. Then the best competitive ratio is exactly $2 k-1$. This is achieved for $K_{1, k}$, the so-called star metric space, where the weights are all ones.

The randomized setup for the above on-line game is the following: first, $A$ has to construct $S$ and $R$ in advance and disclose $S$. Then $A$ gives the points of $R$, one by one, but this time he has no right to make any changes in the requests, no matter how $B$ is playing. That is, not only $R$ but the ordering in which the points of it are requested are determined in advance. In this setup $B$ has the advantage of using randomness when deciding which point of $S$ to be matched with the newly requested point. Let $\operatorname{opt}(\rho)$ be the total weight of the optimum matching for a sequence of requests $\rho$. We say that $B$ 's randomized strategy is $c$-competitive if for every request sequence $\rho$

$$
E[B(\rho)] \leq c \cdot \operatorname{opt}(\rho),
$$

where $E[B(\rho)]$ denotes the expected total weight of the matching $B$ finds for $\rho$. Finding good randomized algorithms for the on-line minimum matching problem was first addressed by Kalyanasundaram and Pruhs in [8]. They stated that the optimal competitive ratio for a star metric space is $2 H_{k}-1$, and conjectured an $O(\log n)$ upper bound on the best competitive ratio for arbitrary metric spaces. Here and later $n$ stands for the number of servers (or requests).

Our goal is to show the following theorem.

Theorem 1 There is a randomized on-line weighted matching algorithm for arbitrary metric spaces which is $O\left(\log ^{3} n / \log \log n\right)$-competitive against an oblivious adversary.

The strategy of the proof is the following. First we show that it is enough to consider the case when the metric space $M$ is a finite space, indeed $X$ is the set of servers. This will cost only a constant factor of at most 3 . Then we develop a randomized weighted greedy matching algorithm (RWGM) that has competitive ratio $O(\log n)$ if the points of $M$ are the leaves of a hierarchically well separated tree, or HST. Here the distance $d(x, y)$ is defined by adding up the weights on the edges of the unique paths connecting $x$ and $y$, and the edge weights grow exponentially by the levels of the tree. In our case the smallest weights are of size $O(\log n)$. In order to use this special case, we recall earlier results on probabilistically approximating arbitrary metric spaces by such trees next. This approximation contributes a $O\left(\log ^{2} n / \log \log n\right)$ factor to the competitive ratio, so finally we arrive at an algorithm with competitive ratio $O\left(\log ^{3} n / \log \log n\right)$.

Independently of this work Meyerson, Nanavati and Poplawski [13] exhibited a randomized on-line algorithm for the matching problem. They also proved a polylog competitive ratio, and used $H S T$ s.

\section{Discretizing the game}

Assume that we have an on-line matching algorithm $M A$ that is $c$-competitive in the possibly infinite metric space $M$ in case $R \subset S$ (multiplicities allowed). In this 
subsection we will show that with a small loss in the competitive factor, $M A$ can easily be extended to an on-line matching algorithm $M A I$ which works for arbitrary $S, R \subset M$. The extension of the algorithm is based on a transformation of $R$ which we call discretization.

Given $S$ assume that the elements of $R$ appear one after the other. For $r_{i} \in R$ we assign a new point $g\left(r_{i}\right) \in S$. We determine $g\left(r_{i}\right)$ in a greedy fashion: if $d\left(s_{0}, r_{i}\right)=$ $\min _{s \in S} d\left(s, r_{i}\right)$, then $g\left(r_{i}\right)=s_{0}$ (breaking ties arbitrarily). Clearly, we can find $g\left(r_{i}\right)$ on-line. For $s \in S$ denote $r m_{s}$ the number of requests which are assigned to $s$ by $g$. The new multiset of requests is called $R^{\prime}$, in which every $s \in S$ appears $r m_{s}$ times. $R^{\prime}$ is the discretized version of $R$.

As above, assume that $M A$ is a $c$-competitive on-line algorithm in the case where $R \subset S$. Clearly, after the discretization we arrive at an $R^{\prime}$ such that $R^{\prime} \subset S$. We give another on-line algorithm $M A I$ in the following way: we play another, auxiliary on-line matching game on $M$ using $M A$, and use $M A$ 's decisions to determine which server $M A I$ chooses to serve a request. Suppose that a request $r \in R$ appears. We determine $g(r)$, and serve this request using the server returned by $M A$. If $M A$ chooses $s \in S$ to serve $g(r)$, then $M A I$ will serve $r$ using $s$.

Lemma 2 If $M A$ is c-competitive, then $M A I$ is $(2 c+1)$-competitive for arbitrary $S, R \subset M$.

Proof: We start with some more notation. For a matching algorithm $A$ denote $A\left(r_{i}\right)$ the distance from $r_{i}$ to $s$ if $A$ serves this request using $s$. Denote $O M$ the optimal cost matching between $S$ and $R$, and let opt $=\operatorname{cost}(O M) . O M$ induces a matching $O M^{\prime}$ (not necessarily of minimum cost) between $S$ and $R^{\prime}$ in the obvious way: if $\left(r_{i}, s_{j}\right) \in$ $O M$, then $\left(g\left(r_{i}\right), s_{j}\right) \in O M^{\prime}$. For an arbitrary matching $M, M\left(r_{i}\right)=d\left(r_{i}, s_{j}\right)$ if $\left(r_{i}, s_{j}\right) \in M$. Finally, let us denote by $o p t^{\prime}$ the total cost of the minimum matching between $S$ and $R^{\prime}$.

From a trivial lower bound on the optimum and by the repeated use of the triangle inequality we have $\sum_{i=1}^{n} d\left(r_{i}, g\left(r_{i}\right)\right) \leq$ opt. Note that $\operatorname{cost}\left(O M^{\prime}\right) \geq o p t^{\prime}$ by definition. By the triangle inequality $M A I\left(r_{i}\right) \leq M A\left(g\left(r_{i}\right)\right)+d\left(g\left(r_{i}\right), r_{i}\right)$, hence, $\sum_{i=1}^{n} M A I\left(r_{i}\right) \leq \sum_{i=1}^{n} M A\left(g\left(r_{i}\right)\right)+$ opt.

Again by the triangle inequality, $\operatorname{cost}\left(O M^{\prime}\left(g\left(r_{i}\right)\right)\right) \leq \operatorname{cost}\left(O M\left(r_{i}\right)\right)+d\left(g\left(r_{i}\right), r_{i}\right)$ for all $i=1, \ldots n$, that sum up to $\operatorname{cost}\left(O M^{\prime}\right) \leq \operatorname{cost}(O M)+\sum_{i=1}^{n} d\left(g\left(r_{i}\right), r_{i}\right)$. That is

$$
o p t^{\prime} \leq \operatorname{cost}\left(O M^{\prime}\right) \leq \operatorname{cost}(O M)+\sum_{i=1}^{n} d\left(r_{i}, g\left(r_{i}\right)\right) \leq \operatorname{cost}(O M)+o p t \leq 2 o p t .
$$

$M A$ is a $c$-competitive on-line algorithm by assumption, i. e., $\sum_{i=1}^{n} M A\left(r_{i}\right) \leq c \cdot o p t^{\prime}$. We know that $M A I\left(r_{i}\right) \leq M A\left(r_{i}\right)+o p t\left(r_{i}\right)$, therefore, $\sum_{i=1}^{n} M A I\left(r_{i}\right) \leq c \cdot o p t^{\prime}+o p t \leq$ $(2 c+1)$ opt.

Remark. Lemma 2 gives an alternative proof of the theorem of Kalyanasundaram and Pruhs [7], that the competitive ratio of the greedy algorithm is at most $2^{n}-1$. Indeed, let $M A$ and $M A I$ be the greedy algorithms for an $n-1$ and an $n$ element input, respectively, and use induction. 


\section{The algorithm RWGM}

Our algorithm, the randomized weighted greedy matching algorithm, or RWGM algorithm is first developed for special metric spaces. Assume that the metric space $M=(X, d)$ is defined by a weighted tree $T$. The set of the leaves of $T$ is $L \subset X$, and the distance $d(x, y)$ for the leaves $x, y$ is the sum of the weights on the (unique) path connecting $x$ and $y$. Let $\lambda>1$ be a real number.

Definition 1 A $\lambda$-hierarchically well separated tree $(\lambda-H S T)$ is a rooted weighted tree with the following properties:

- the edge weight from any node to each of its children is the same,

- the edge weights along any path from the root to a leaf are decreasing by the factor $\lambda$ from one level to the next. The weight of an edge incident to a leaf is one.

We define the RWGM algorithm first, then show in steps that it is $O(\log n)$-competitive on a metric space determined by a $\lambda$-HST where $\lambda=2(1+\log n)$.

\subsection{RWGM: a randomized weighted matching algorithm for hierar- chically well separated trees}

Let us consider a $\lambda$-HST, denote it by $T=T(V, E, r)$, where $V$ is the vertex set, $E$ is the edge set of $T$, and $r$ is the root. When playing the matching game only leaves of $T$ will be matched to leaves of $T$. We denote the set of leaves by $L$. We will need the notion of a subtree: given $v \in V$, the vertex $u \in V$ belongs to the subtree $T_{v}$ if the only path from $r$ to $u$ contains $v$. Clearly, $T=T_{r}$, and if $w \in L$, then $T_{w}$ contains only the leaf $w$. We have the relation " $\leq$ " among the subtrees containing a certain leaf $w: T_{u} \leq T_{u^{\prime}}$ if $\left|T_{u}\right| \leq\left|T_{u^{\prime}}\right|, w \in T_{u}, w \in T_{u^{\prime}}$.

In order to get an easier formulation of RWGM, we assume that if $u$ is a non-leaf vertex of a $(\log n)$-HST, then all of its children are non-leaves or all are leaves. This can be achieved by inserting "dummy" vertices in the tree. We can also assume that the edge weights on a level are equal. (See [5].)

During the course of satisfaction of the requests, certain vertices will be painted green, and leaves may have multiplicities. A vertex $x$ is green if the subtree $T_{x}$ contains at least one unassigned server. We need multiplicities since a point (as a server) may be listed with multiplicity, and also it may be requested several times. (Recall from the introduction that $S$ and $R$ are multisets of $V(G)$.) The colors and multiplicities of the vertices may change in time, as we satisfy the requests and using up the servers. We try to follow the greedy algorithm, and break ties by random selection by levels.

Informally, having a request $r$, we try to assign to $r$ a server $s$ as close as possible according to the tree-metric. One visualizes this as going up in $T$ until reaching the first green vertex $x$, and then going down to an unassigned server. However, going down from $x$ is unintuitive: we choose uniformly among those edges $\left(x, y_{1}\right), \ldots,\left(x, y_{k}\right)$ that lead to unassigned servers. One is tempted to go down on $(x, y)$ with probability proportional to the number of unassigned servers in $T_{y}$. This other approach is analyzed in [13]. 


\section{Formal description of RWGM}

In the beginning the adversary $A$ picks leaves of $T$ with multiplicity, corresponding to the servers $S=s_{1}, \ldots, s_{n}$. (That is if a leaf $x$ is provided $m$ times as a server then $x$ has multiplicity $m$.)

We color a vertex $u$ of $T$ green if $T_{u}$ contains a leaf with positive multiplicity, and will call such subtrees green subtrees.

Then $A$ will give us the requests of $R$ one-by-one, denote them by $r_{1}, \ldots, r_{n}$.

Set $i=1$.

- Step 1. The new request is $r_{i}$. $B$ looks for the smallest subtree $T_{u}$ which contains $r_{i}$, and $u$ is green.

- Step 2. Pick a leaf of $T_{u}$ among the leaves of positive multiplicity by the algorithm Pick-a-leaf with input $u$. Let this leaf be $x$, and let $s_{i}$ (perhaps after reordering) be an unused server corresponds to that is matched to $r_{i}$. Decrease the multiplicity of $x$ by one.

- Step 3. For every green $w \in V$ check whether $T_{w}$ contains a leaf with positive multiplicity. If not, erase $w$ 's color.

- Step 4. If $i \leq n-1$, then set $i=i+1$, then go to Step 1 .

- Step 5. If $i=n$, then STOP.

\section{Algorithm Pick-a-leaf $(u)$}

- Step 1. If the children of $u$ are leaves, then pick randomly, uniformly a leaf among those of positive multiplicity. This is the leaf we have chosen. STOP.

- Step 2. If the children of $u$ are not leaves, then denote $u_{1}, u_{2}, \ldots, u_{t}$ the green children of $u$. Pick one randomly, uniformly among them, say, it is $u_{i}$. Apply Pick-a-leaf $\left(u_{i}\right)$.

Theorem 3 The algorithm RWGM is $O(\log n)$-competitive on a metric space determined by a $\lambda-H S T$ against an oblivious adversary.

\subsection{Proof of Theorem 3}

We prove Theorem 3 in steps. First we consider the case of uniform metric space where the multiplicities are all ones, but the sizes of $S$ and $R$ may not be equal. Then we discuss the case where $S$ and $R$ have arbitrary multiplicities. Finally we prove the general statement for HST's; here the previous cases provide a basis for induction arguments. 


\subsubsection{Uniform case}

In a uniform metric space the distance of two different points is one. It closely resembles to the star metric space $K_{1, k}$, where the leaves are of a distance two from each other. (This explains the extra two factor in some of our later formulas.)

Assume that $U$ is the uniform metric space on $u$ points. Let $S=\left\{s_{1}, \ldots, s_{q}\right\}$ and $R=\left\{r_{1}, \ldots, r_{t}\right\}, s_{i} \neq s_{j}$ and $r_{i} \neq r_{j}$ if $i \neq j$. We also assume that the points of $R$ are requested in increasing order, first $r_{1}$, then $r_{2}$, etc., and finally $r_{t}$.

Before dealing with the general case, let us consider a simple but instructive example, when $|S|=|R|=q$, and these sets share $q-1$ points. Clearly, the worst case if the first request $r_{1}$ is not in $S$. Assigning $r_{1}$ to some $s_{i}$ for which there is an $r_{j}=s_{i}$ destroys optimality. This mistake may spread when we match $r_{j}$. It was noted in [8] that any randomized on-line algorithm for that instance has about $\log q$ expected cost, although the optimal cost is one. This explains why we have to take special care of the common points of $S$ and $R$, and also the order of requests.

Definition 2 We say that $s_{i} \in S$ has a partner if $s_{i}=r_{j}$ for some $r_{j} \in R$. Similarly, $r_{j} \in R$ has a partner if $s_{i}=r_{j}$ for some $s_{i} \in S$.

We will give an ordering of the points of $S$ using the above mentioned ordering on $R$. Firstly if there exist $r_{j}$ and $r_{\ell}$ such that $s_{i}$ is the partner of $r_{j}$ and $s_{k}$ is the partner of $r_{\ell}$ where $j<\ell$, then $s_{i}<s_{k}$. If $s_{i}$ has a partner and $s_{k}$ has no partner, then $s_{i}<s_{k}$ and $r_{j}<s_{k}$ for all $j$. Finally, we fix an arbitrary ordering among those points of $S$ which have no partner in $R$. Notice, that we can extend the orderings of $S$ and $R$ into an ordering "<" of $S \cup R$. This is done such that if $r_{i}$ is the partner of $s_{j}$ then $r_{i}<s_{j}$, and for $r_{k}>r_{i}$ we have $s_{j}<r_{k}$. The points of $S$ having no partner go to the end of the ordering.

Given $r_{i} \in R$ we associate a weight $w\left(r_{i}\right)$ with it. It is the difference of the number of servers following, and the number of requests without partner preceeding $r_{i}$. Let us assume that $r_{i}$ has no partner, then

$$
v_{i}=\left|\left\{s_{j}: s_{j}>r_{i}\right\}\right|-\mid\left\{r_{k}: r_{k}<r_{i} \text { and } r_{k} \text { has no partner }\right\} \mid .
$$

If $r_{i}$ has a partner, then let $v_{i}=0$. Furthermore let $H_{m}=1+\frac{1}{2}+\ldots+\frac{1}{m}$, that is the $m^{\text {th }}$ Harmonic number. Then we define $w\left(r_{i}\right)=H_{v_{i}}$ (we let $H_{f}=0$ if $f \leq 0$ ). We need the following useful lemma.

Lemma 4 For $n \geq 1, H_{n}=1+\frac{H_{n-1}+\ldots+H_{1}}{n}$.

Proof: Trivial computation.

Lemma 5 Let $\delta=|R-S|$. Then in the case above the expected cost of RWGM is at most $H_{q}+H_{q-1}+\ldots+H_{q-\delta+1}$.

Proof: We proceed by induction on $q$ that is the size of $S$. Notice that we may assume that $r_{1}$ has no partner, otherwise we can immediately apply the induction hypothesis. Now $r_{1}$ is matched to some randomly chosen $s_{j} \in S$. One can check by 
the definition of $v_{i}$ that the weights of the elements of $R \backslash\left\{r_{1}\right\}$ are invariant if $s_{j}$ had no partner. If $s_{j}$ had the partner $r_{i}$ then the expected new weight of $r_{i}$ is at most $\left(H_{q-1}+\ldots+H_{1}\right) / q$. Now by induction one can see that for the resulting smaller subproblem the random algorithm has expected cost $H_{q-1}+\ldots+H_{q-\delta+1}$. Match of $r_{1}$ to $s_{j}$ costs one, hence, the expected cost of the algorithm is at most

$$
1+\frac{H_{q-1}+\ldots+H_{1}}{q}+H_{q-1}+\ldots+H_{q-\delta+1}=H_{q}+H_{q-1}+\ldots+H_{q-\delta+1},
$$

by Lemma 4 .

\subsubsection{The case of multiplicities}

We want to handle the case when both the servers and the requests have various multiplicities. Note, that a server with zero multiplicity simply means that there is no server at that point. If $U=x_{1}, \ldots, x_{u}$, then let $m s\left(x_{i}\right)$ and $m r\left(x_{j}\right)$ are the multiplicities of servers and requests in point $x_{i}$ and $x_{j}$, respectively. Let $\delta\left(x_{i}\right)=$ $\max \left\{0, m r\left(x_{i}\right)-m s\left(x_{i}\right)\right\}, \delta=\sum_{i=1}^{u} \delta\left(x_{i}\right)$.

Lemma 6 The expected cost of $R W G M$ is at most $H_{q}+H_{q-1}+\ldots+H_{q-\delta+1}$.

Proof: Fix a maximum matching between servers at requests which belong to the same point. Pretend that the remaining unmatched equal servers/requests are at different points, and apply Lemma 5 .

\subsubsection{General $\lambda$-HST trees}

We proceed by induction on the height of the $\lambda$-HST tree. First, we need a more technical form of the hypothesis and some definitions.

Definition 3 Given $s \in S$ and $r \in R$, which are matched in some matching $M$, consider the path connecting them in the HST tree. Call the point at the highest level of this path the turning point of $s$ and $r$, shortly $t_{M}(s, r)$. For a point $u$ of the tree let $\tau_{M}(u)$ be the number of $(s, r)$ matched pairs in $M$ for which $u$ is a turning point.

Given a point $u, h(u)$ will denote the height of $u$. We can express the cost of an arbitrary matching $M$ as

$$
2 \sum_{u} \tau_{M}(u) \sum_{i=1}^{h(u)} \lambda^{i-1} .
$$

Observe that $\tau_{M}(u)$ is the same for any optimal matching $M$, hence in this case we suppress the subscript $M$. Note that $\tau(u)$ is obvious to compute. Moreover, one can express the optimal cost:

$$
\text { opt }=2 \sum_{u} \tau(u) \sum_{i=1}^{h(u)} \lambda^{i-1} .
$$

For trees of height less than $d$ our induction hypothesis is the following inequality: 


$$
\mathbf{E}[\mathrm{RWGM}] \leq 2 \sum_{u} \tau(u) \sum_{i=1}^{h(u)} c_{i} \lambda^{i},
$$

where $\lambda=2(1+\log n), c_{1}=1 / 2$ and $c_{t}:=c_{t-1}+(1 / 2)^{t}$ for $t>1$. Notice, that since $c_{t} \leq 1$, the above statement proves that RWGM is $O(\log n)$-competitive against an oblivious adversary implying Theorem 3 .

For trees of height one the statement follows from Lemma 6 and its remark. Consider a tree $T$ of height $d$. We make a new tree $T^{\prime}$ and a new instance $S^{\prime}$ and $R^{\prime} . T^{\prime}$ comes from $T$ by pruning the leaves, and for a $u \in T, h(u)=1$ we associate the server and request multiplicities that of the sum of the server and request multiplicities of its descendants in $T . T^{*}$ denotes the set of subtrees of $T$ of height one, i. e. the leaves and their parents. Note that we have to divide the edge weights of $T^{\prime}$ by $\lambda$ in order to get a $\lambda$-HST-tree.

One can cut the optimal cost for $S, R$ and $T$ in two parts. The first part is the optimal cost of $S^{\prime}, R^{\prime}$ and $T^{\prime}$, which we call opt ${ }^{\prime}$. The second part is the cost incurring on $T^{*}$, this is opt* Here we have to take care of cases when the number of requests are greater than the number of servers in a subtree $T_{u}(h(u)=1)$. Then we consider the partial optimal matching using those servers. Let us call the cost of this partial matching, opt $t_{u}^{*}$ the optimal for this case.

Clearly, opt ${ }^{*}=\sum_{u: h(u)=1} \mathrm{opt}_{u}^{*}=\sum_{u: h(u)=1} 2 \tau(u)$ and one concludes that

$$
\text { opt }=\lambda \cdot \mathrm{opt}^{\prime}+\sum_{u: h(u) \geq 2} 2 \tau(u)+\mathrm{opt}^{*} .
$$

Unfortunately, the on-line cost on $T$ is not the sum of the on-line costs of the two parts if we handle the parts separately, but they are closely related.

For this reason we have to take care of the costs occurring in $T^{*}$ when such a request is assigned to a leaf of a tree $T_{u}$ which is not supposed in the optimal matching. The exact form of this statement is spelled out in Lemma 7.

Let $\mathcal{M}$ be a random matching resulted from the run of RWGM on our tree. Then $\tau_{\mathcal{M}}(u)$ is a random variable for each $u$ non-leaf, and $M=\sum_{u} \tau_{\mathcal{M}}(u)$ is a random variable again.

\section{Lemma 7}

$$
\mathbf{E}[M] \leq \sum_{u: h(u) \geq 1} \tau(u) \sum_{i=1}^{h(u)}(1+\log n)^{i} .
$$

Proof: We prove Lemma 7 by induction on the height of the tree. It is true for trees of height one by Lemma 6. Assuming that the lemma is true for trees of height at most $h$, we will show it for trees of height $h+1$.

Let $T$ be a $\lambda$-HST tree of height $h+1$. We define $T^{\prime}$ and $T^{*}$ as before. $M^{\prime}$ is just the truncated sum of $M$ on $T^{\prime}$. By the induction hypothesis we have the following inequality: 


$$
\mathbf{E}\left[M^{\prime}\right] \leq \sum_{u: h(u) \geq 2} \tau(u) \sum_{i=1}^{h(u)-1}(1+\log n)^{i} .
$$

Note furthermore that every extra request arriving from $T^{\prime}$ to a vertex $u$ of height one (i. e. to a root of a tree $T_{u}$ of the forest $T^{*}$ ) increases the expected cost of RWGM in $T_{u}$ by at most $\log n$ by Lemma 6 .

The average cost on the trees of $T^{*}$ comes from two sources; one is opt $t^{*}$, the other is $M^{\prime}$. In order to get an upper bound on the cost on $T^{*}$ we have to add them up and multiply both by $\log n$, according to the explanation in the previous paragraph. This way we have

$$
\begin{gathered}
\mathbf{E}[M] \leq \log n\left\{\sum_{u: h(u)=1} \tau(u)+\mathbf{E}\left[M^{\prime}\right]\right\}+\mathbf{E}\left[M^{\prime}\right]= \\
\sum_{u: h(u)=1} \tau(u) \log n+\sum_{u: h(u) \geq 2} \tau(u) \sum_{i=2}^{h(u)}(1+\log n)^{i} \leq \sum_{u: h(u) \geq 1} \tau(u) \sum_{i=1}^{h(u)}(1+\log n)^{i},
\end{gathered}
$$

which proves the lemma.

Now we will use this lemma to prove that

$$
\mathbf{E}[\mathrm{RWGM}] \leq 2 \sum_{u} \tau(u) \sum_{i=1}^{h(u)} c_{i} \lambda^{i} .
$$

Again we will proceed by induction. Assume that the statement is true for trees of height at most $h$, and consider a tree $T$ of height $h+1$. We prune the leaves of $T$, thereby getting $T^{\prime}$. Recall, that edge weights in $T$ has to be divided by $\lambda$ so as to get that $T^{\prime}$ is a $\lambda$-HST. For $T^{\prime}$ the statement is true by the induction hypothesis. That is, the expected cost of RWGM on $T^{\prime}$ is at most

$$
\mathbf{E}\left[\operatorname{RWGM}\left(T^{\prime}\right)\right] \leq 2 \sum_{u: h(u) \geq 2} \tau(u) \sum_{i=1}^{h(u)-1} c_{i} \lambda^{i} .
$$

Clearly, if we add this up with the expected cost at level one, we get an upper bound for the expected cost of RWGM on $T$ :

$$
\mathbf{E}[\operatorname{RWGM}(T)] \leq \lambda \cdot \mathbf{E}\left[\operatorname{RWGM}\left(T^{\prime}\right)\right]+2 \cdot \mathbf{E}[M] .
$$

We want to show that this is at most

$$
2 \sum_{u} \tau(u) \sum_{i=1}^{h(u)} c_{i} \lambda^{i}
$$

The coefficient of $\tau(u)$ in the upper bound is less than $\sum_{i=1}^{h(u)} c_{i} \lambda^{i}$ for every $u$ at level $\ell$. For $\ell=1$, it follows since $\log n \leq c_{1} 2(1+\log n)$. 
For $\ell>1$, we need to show that

$$
\log n \sum_{i=1}^{\ell-1}(1+\log n)^{i}+\sum_{i=1}^{\ell-1} c_{i} \lambda^{i+1} \leq \sum_{i=1}^{\ell} c_{i} \lambda^{i} .
$$

It follows if

$$
\log n \sum_{i=1}^{\ell-1}(1+\log n)^{i} \leq \sum_{i=2}^{\ell}\left(c_{i}-c_{i-1}\right) \lambda^{i}+c_{1} \lambda .
$$

Since $\log n \sum_{i=1}^{\ell-1}(1+\log n)^{i}=(1+\log n)^{\ell}-(1+\log n)$, it reduces to

$$
(1+\log n)^{\ell} \leq \sum_{i=1}^{\ell}\left(\frac{1}{2}\right)^{i}(2+2 \log n)^{i}=\sum_{i=1}^{\ell}(1+\log n)^{i} .
$$

\section{Approximating by hierarchically well separated trees}

The first results and applications of hierarchically well separated trees are due to Bartal, see in [2, 3]. It generalized the earlier works of Karp [10] and Alon et al [1] in which they approximated the distances in certain graphs by using randomly selected spanning trees.

Definition $4 A$ metric space $M=\left(X, d_{M}\right)$ dominates a metric space $N=\left(X, d_{N}\right)$ if for every $x, y \in X$ we have $d_{N}(x, y) \leq d_{M}(x, y)$.

Definition $5 A$ set of metric spaces $S$ over $X$-probabilistically approximates a metric space $M$ over $X$, if every metric space in $S$ dominates $M$, and there exists a probability distribution over metric spaces $N \in S$ such that for every $x, y \in X$ we have $E\left[d_{N}(x, y)\right] \leq \alpha d_{M}(x, y)$.

The proof of Theorem 1 is based on the following theorem.

Theorem 8 [5] Every weighted graph on $n$ vertices can be $\alpha$-probabilistically approximated by a set of $\lambda$-HSTs, for an arbitrary $\lambda>1$ where $\alpha=O(\lambda \log n / \log \lambda)$.

As noted by Bartal [2], having an approximation of a metric space $M$ by HST trees along with a good algorithm for such trees always results in a good randomized algorithm in that space. So, what we do is the following. First, preprocessing: given the set of servers $S$, these points span a sub-metric space $\mathcal{M}_{\mathcal{S}} \subset \mathcal{M}$. Clearly, $\left|\mathcal{M}_{\mathcal{S}}\right| \leq$ $n$, since $S$ is a multiset of $n$ elements. We approximate $\mathcal{M}_{\mathcal{S}}$ by a set of $O(\log n)$-HSTs. Plugging in $\lambda=2(1+\log n)$ into Theorem 8 we get there is a probability distribution $\mathcal{P}$ on these trees such that the expected distortion is $O\left(\log ^{2} n / \log \log n\right)$. Choose one tree at random according to $\mathcal{P}$. This finishes the preprocessing. Whenever a request $r \in R$ appears, we determine $g(r)$ (see Section 2), and use RWGM with this new request $g(r)$. We proved in Section 3, that RWGM is a $O(\log n)$-competitive algorithm in this case. Applying Lemma 2 and Theorem 8, we get that RWGM is $O\left(\log ^{3} n / \log \log n\right)$ competitive for $\mathcal{M}$. This proves Theorem 1 . 
Acknowledgment. We thank Endre Szemerédi and Kirk Pruhs for the fruitful discussions. The numerous advices of anonymous referees also improved a lot on the presentation of the paper.

\section{References}

[1] N. Alon, R. M. Karp, D. Peleg, D. West, A graph-theoretic game and its application to the $k$-server problem, SIAM J. Comput. 24 (1) (1995) 78-100.

[2] Y. Bartal, Probabilistic approximations of metric spaces and its algorithmic applications, in: IEEE Symposium on Foundations of Computer Science, 1996, pp. 184-193.

[3] Y. Bartal, On approximating arbitrary metrics by tree metrics, in: STOC, 1998.

[4] Y. Bartal, M. Charikar and R. Raz, Approximating min-sum k-clustering in metric spaces, Thirty-Third Annual ACM Symposium on Theory of Computing, pages 11-20, 2001.

[5] J. Fakcharoenphol, S. Rao and K. Talwar, A tight bound on approximating arbitrary metrics by tree metrics, J. Comput. System Sci. 69 (2004), no. 3, $485-497$.

[6] B. Fuchs, W. Hochstättler, and W. Kern. Online matching on a line, In Hajo Broersma, Ulrich Faigle, Johann Hurink, Stefan Pickl, and Gerhard Woeginger, editors, Electronic Notes in Discrete Mathematics, volume 13. Elsevier, 2003.

[7] B. Kalyanasundaram and K. Pruhs, Online weighted matching, Journal of Algorithms, 14(3) (1993) 478-488.

[8] B. Kalyanasundaram, K. Pruhs, On-line network optimization problems, in $O n$ line algorithms: The State of the Art, eds. A. Fiat and G. Woeginger, pages 268-280 Lecture Notes in Comput. Sci., 1442, Springer, Berlin, (1998)

[9] B. Kalyanasundaram, K. Pruhs, The online transportation problem, SIAM J. Discrete Math. 13 (2000), no. 3, 370-383.

[10] R. Karp, A 2k-competitive algorithm for the circle. Manuscript, August 1989.

[11] S. Khuller, S. G. Mitchell, V. V. Vazirani, On-line algorithms for weighted bipartite matching and stable marriages, Theoret. Comput. Sci. 127 (1994), no. $2,255-267$.

[12] E. Koutsoupias, A. Nanavati, The online matching problem on a line, Approximation and online algorithms, 179-191, Lecture Notes in Comput. Sci., 2909, Springer, Berlin, 2004.

[13] A. Meyerson, A. Nanavati, L. Poplawski, Randomized on-line algorithms for minimum metric bipartite matching, SODA (2006). 
[14] Y. T. Tsai, C. Y. Tang, Y. Y. Chen, Average performance of a greedy algorithm for the on-line minimum matching problem on Euclidean space, Inform. Process. Lett. 51 (1994), no. 6, 275-282. 